\title{
Efeito das condições operacionais e estudo cinético na degradação eletroquímica do corante azul de metileno sobre anodo de $\mathrm{Ti} / \mathrm{Ru}_{0.3} \mathrm{Ti}_{0.7} \mathrm{O}_{2}$
}

\author{
Effect of operational conditions and kinetic study in the electrochemical degradation of methylene \\ blue dye onto a $\mathrm{Ti} / \mathrm{Ru}_{0.3} \mathrm{Ti}_{0.7} \mathrm{O}_{2}$ anode \\ Efecto de las condiciones de operación y el estudio cinético en la degradación electroquímica del \\ colorante azul de metileno utilizando ánodo $\mathrm{Ti} / \mathrm{Ru}_{0.3} \mathrm{Ti}_{0.7} \mathrm{O}_{2}$
}

Recebido: 10/04/2021 | Revisado: 18/04/2021 | Aceito: 24/04/2021 | Publicado: 07/05/2021

Tobias de Oliveira Souza

ORCID: https://orcid.org/0000-0003-0477-0726 Universidade Estadual do Ceará, Brasil E-mail: tobiasvasconcelos1@gmail.com

Jackson Anderson Sena Ribeiro

ORCID: https://orcid.org/0000-0003-0704-2927 Instituto Federal de Educação, Ciência e Tecnologia do Ceará, Brasil E-mail: jacksonsenaribeiro@gmail.com

Francisco Everardo Pereira Viana

ORCID: https://orcid.org/0000-0003-0365-2285 Instituto Federal de Educação, Ciência e Tecnologia do Ceará, Brasil E-mail: everardopereira@live.com

Jéssica Rocha de Lima

ORCID: https://orcid.org/0000-0002-9297-9660 Instituto Federal de Educação, Ciência e Tecnologia do Ceará, Brasil E-mail: jehrocha12@gmail.com

Janaina de Vasconcelos Cruz ORCID: https://orcid.org/0000-0002-1198-9956 Instituto Federal de Educação, Ciência e Tecnologia do Ceará, Brasil E-mail: janaina@ifce.edu.br

Edson da Silva Almeida

ORCID: https://orcid.org/0000-0001-7510-6983 Instituto Federal de Educação, Ciência e Tecnologia do Ceará, Brasil E-mail: edson@ifce.edu.br

Rinaldo dos Santos Araújo ORCID: https://orcid.org/0000-0003-2609-436X Instituto Federal de Educação, Ciência e Tecnologia do Ceará, Brasil E-mail: rinaldo@ifce.du.br

\begin{abstract}
Resumo
Efluentes têxteis e alimentícios apresentam comumente em sua composição compostos corantes danosos à saúde humana e ao meio ambiente e neste contexto tecnologias de oxidação avançada como os processos eletroquímicos têm despontado como alternativa de tratamento de compostos refratários presentes nestas águas residuárias. Assim, este trabalho investigou a degradação do corante básico azul de metileno a temperatura ambiente $\left(25^{\circ} \mathrm{C}\right)$ via tratamento eletrocatalítico com anodo comercial de $\mathrm{Ti} / \mathrm{Ru}_{0.3} \mathrm{Ti}_{0.7} \mathrm{O}_{2}\left(30 \%\right.$ de $\mathrm{RuO}_{2}$ e $70 \%$ de $\left.\mathrm{TiO}_{2}\right)$. Os estudos experimentais a $\mathrm{pH}=6,8$ foram executados para a avaliação do efeito do potencial de eletrólise, natureza e concentração do suporte eletrolítico e concentração inicial de corante sobre a eficiência e cinética de degradação do corante. Os resultados mostraram degradações superiores a $90 \%$ em $60 \mathrm{~min}$ de tratamento por eletrólise indireta usando $0,01 \mathrm{~mol} \mathrm{~L}^{-1} \mathrm{NaCl}$ e $0,01 \mathrm{~mol} \mathrm{~L}^{-1} \mathrm{Na}_{2} \mathrm{SO}_{4}$ como suporte eletrolítico sob potencial de $5,0 \mathrm{~V}$ para concentrações entre $5 \mathrm{mg} \mathrm{L}^{-1} \mathrm{e}^{-1} \mathrm{mg} \mathrm{L}^{-1}$. A cinética de eletrodegradação foi tipicamente de primeira ordem. Em geral, os valores de eficiência de degradação encontrados ratificaram o caráter promissor da aplicação de anodos dimensionalmente estáveis na despoluição de águas residuárias coloridas.
\end{abstract}

Palavras-chave: Eletrooxidação; Azul de metileno; Anodo Ti/ $\mathrm{Ru}_{0.3} \mathrm{Ti}_{0.7} \mathrm{O}_{2}$; Cinética.

\begin{abstract}
Textile and food effluents commonly contain in their composition dye compounds harmful to human health and the environment and in this context advanced oxidation technologies such as electrochemical processes have emerged as an alternative treatment for refractory compounds present in these wastewaters. Thus, this work investigated the degradation of the basic methylene blue dye at room temperature $\left(25^{\circ} \mathrm{C}\right)$ via electrocatalytic treatment with
\end{abstract}


commercial $\mathrm{Ti} / \mathrm{Ru}_{0.3} \mathrm{Ti}_{0.7} \mathrm{O}_{2}$ anode $\left(30 \% \mathrm{RuO}_{2}\right.$ and $70 \%$ of $\left.\mathrm{TiO}_{2}\right)$. Experimental studies at $\mathrm{pH}=6.8$ were performed to evaluate the effect of the electrolysis potential, nature and concentration of the electrolytic support and initial concentration of dye on the efficiency and kinetics of degradation. The results showed degradations greater than $90 \%$ in $60 \mathrm{~min}$ of treatment by indirect electrolysis using $0.01 \mathrm{~mol} \mathrm{~L}^{-1} \mathrm{NaCl}$ and $0.01 \mathrm{~mol} \mathrm{~L}^{-1} \mathrm{Na}_{2} \mathrm{SO}_{4}$ as electrolytic support under $5.0 \mathrm{~V}$ potential at concentrations between $5 \mathrm{mg} \mathrm{L}^{-1}$ and $25 \mathrm{mg} \mathrm{L}^{-1}$. Electrodegradation kinetics were typically first order. In general, the degradation efficiency values found confirmed the promising character of the application of dimensionally stable anodes in the depollution of colored wastewater.

Keywords: Electrooxidation; Methylene Blue; $\mathrm{Ti} / \mathrm{Ru}_{0.3} \mathrm{Ti}_{0.7} \mathrm{O}_{2}$ anode; Kinetic.

\section{Resumen}

Los efluentes textiles y alimentarios tienen en su composición compuestos colorantes nocivos para la salud humana y el medio ambiente y en este contexto han surgido tecnologías avanzadas de oxidación mediante procesos electroquímicos como tratamiento alternativo para los compuestos refractarios presentes en estas aguas residuales. Así, este trabajo investigó la degradación del colorante básico azul de metileno a temperatura ambiente $\left(25{ }^{\circ} \mathrm{C}\right)$ mediante tratamiento electrocatalítico con ánodo comercial $\mathrm{Ti} / \mathrm{Ru}_{0.3} \mathrm{Ti}_{0.7} \mathrm{O}_{2}\left(30 \% \mathrm{RuO}_{2}\right.$ y $\left.70 \% \mathrm{TiO}_{2}\right)$. Se realizaron estudios experimentales a $\mathrm{pH}=6.8$ para evaluar el efecto del potencial de electrólisis, la naturaleza y concentración del soporte electrolítico y la concentración inicial del colorante sobre la eficiencia y la cinética de degradación del azul de metileno. Los resultados mostraron degradaciones mayores al $90 \%$ en 60 min de tratamiento por electrólisis indirecta utilizando $\mathrm{NaCl} 0.01 \mathrm{~mol} \mathrm{~L}^{-1} \mathrm{y} \mathrm{Na}_{2} \mathrm{SO}_{4} 0.01 \mathrm{~mol} \mathrm{~L}^{-1}$ como soporte electrolítico en el potencial de $5.0 \mathrm{~V}$ para concentraciones entre $5 \mathrm{mg} \mathrm{L}^{-1}$ y $25 \mathrm{mg} \mathrm{L}^{-1}$. La cinética de electro-oxidación fue típicamente de primer orden. En general, los valores de eficiencia de degradación encontrados confirmaron el carácter prometedor de la aplicación de ánodos dimensionalmente estables en la descontaminación de aguas residuales coloreadas.

Palabras clave: Electro-oxidación; Azul de metileno; Ánodo $\mathrm{Ti} / \mathrm{Ru}_{0.3} \mathrm{Ti}_{0.7} \mathrm{O}_{2}$; Cinética.

\section{Introdução}

As questões ambientais relacionadas a presença de corantes constituem problema corriqueiro para os setores indústrias que os utilizam, como é caso das indústrias têxteis, farmacêuticas, de alimentos entre outras (Santos et al., 2020; Indu et al., 2014; Gupta et al., 2006). Os efluentes industriais coloridos prejudicam a atividade fotossintética aquática através do bloqueio da luz, além do que muitos corantes se apresentam como moléculas perigosas à saúde humana e à biota aquática devido aos seus elevados efeitos tóxicos e carcinogênicos (Collivignarelli et al., 2019).

As crescentes evidências da degradação do meio ambiente trazem desafios para manter a capacidade de sobrevivência dos ecossistemas e impulsionam o desenvolvimento de novas tecnologias que respeitem e propiciem a preservação ambiental. A qualidade dos recursos hídricos está entre as principais preocupações ambientais, sendo considerada um importante indicador do bem-estar de uma sociedade.

O azul de metileno (CI: 52015), por exemplo, é o corante mais utilizado na indústria, sendo amplamente utilizado como corante sintético por apresentar boa solubilidade e estabilidade de cor (Fadillah et al., 2019, Li et al., 2006). O azul de metileno é um corante básico classificado no grupo de corantes catiônicos comumente usado em aplicações nas áreas da química, biologia, medicina e de tingimento de tecidos (Indu et al., 2014). A exposição a longo prazo a esse corante pode levar a vômitos, náuseas, anemia e hipertensão (Samarghandi et al., 2020).

Muitos trabalhos da literatura relatam o uso de técnicas de oxidação avançada para o tratamento em efluentes têxteis (Oliveira et al., 2020; Yaseen \& Scholz, 2019). Particularmente, em processos eletroquímicos as degradações dos poluentes podem ocorrer via oxidação direta na superfície do anodo, ou indiretamente, quando há a produção no meio de outras espécies químicas (oxidação indireta) capazes de converter os compostos iniciais em espécies menos refratárias (Martínez-Huitle \& Panizza, 2018).

Anodos dimensionalmente estáveis $\left(\mathrm{DSA}^{\circledR}\right)$ denotam uma classe de eletrodos de óxidos preparados termicamente, onde um substrato metálico, por exemplo de titânio, é recoberto por um óxido metálico ativo como $\mathrm{RuO}_{2}, \mathrm{IrO}_{2}, \mathrm{TiO}_{2}\left(\mathrm{Krstić}_{\&}\right.$ Pešovski, 2019). Eletrodos DSA apresentam vantagens como: baixa sobrevoltagem para a evolução de $\mathrm{O}_{2}$ e $\mathrm{Cl}_{2}$, boa estabilidade mecânica, elevadas áreas ativas, baixos custos de manutenção e baixos consumos de energia (Santos et al., 2020). 
Eletrodos revestidos com $\mathrm{RuO}_{2}$ e $\mathrm{IrO}_{2}$ são usados na oxidação indireta com formação no meio reacional de espécie como $\mathrm{Cl}_{2}$, $\mathrm{HOCl}$ e $\mathrm{OCl}$, enquanto $\mathrm{SnO}_{2}, \mathrm{PbO}_{2}$ e BDD são indicados para a oxidação direta via radicais `OH (Cruz-Díaz et al., 2018).

Nesse contexto, este trabalho tem como objetivo avaliar a eficiência e a cinética de eletrodegradação de azul de metileno em meio aquoso sobre um eletrodo de $\mathrm{Ti} / \mathrm{Ru}_{0,3} \mathrm{Ti}_{0,7} \mathrm{O}_{2}\left(\mathrm{DSA}-\mathrm{Cl}_{2}\right)$. Os estudos serão conduzidos para a investigação dos efeitos das variáveis de potencial de eletrólise, natureza/concentração do suporte eletrolítico e concentração inicial de corante sobre a performance do tratamento eletrocatalítico proposto.

\section{Metodologia}

\subsection{Reagentes}

O corante azul de metileno dihidratado PA (Figura 1) foi fornecido pela Isofar Ltda (Brasil). A água ultrapura (condutividade 18,2 $\mathrm{M} \Omega \mathrm{cm}^{-2}$ ) usada no preparo das soluções foi obtida de uma unidade de osmose reversa Purelab. Álcool isopropílico PA (Vetec, Brasil) foi utilizado para o desengraxe da superfície dos eletrodos na etapa de limpeza. O eletrodo de trabalho foi do tipo $\mathrm{Ti} / \mathrm{Ru}_{0.3} \mathrm{Ti}_{0.7} \mathrm{O}_{2}$, o qual foi gentilmente cedido pela De Nora Brasil Ltda, sendo classificado como anodo dimensionalmente estável para uso na indústria de cloro-álcalis (DSA-Cl $)$. $\mathrm{Na}_{2} \mathrm{SO}_{4} \mathrm{e} \mathrm{NaCl}$ utilizados na preparação dos meios eletrolíticos foram fornecidos pela Dinâmica e usados sem qualquer processo de purificação prévia.

Figura 1. Estrutura molecular do corante azul de metileno.

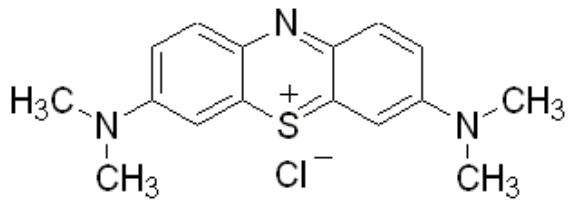

Fonte: Autores.

\subsection{Caracterização do eletrodo}

A caracterização superficial do eletrodo de $\mathrm{Ti} / \mathrm{Ru}_{0.3} \mathrm{Ti}_{0.7} \mathrm{O}_{2}$ foi executada em microscópio eletrônico de varredura PHILIPS modelo XL-30 o qual possui um acoplado um acessório de análise por espectroscopia de energia dispersiva de raiosX (SEM/EDX) modelo LINK Analytical, modelo QX-2000. A caracterização A caracterização voltamétrica foi realizada em um potenciostato PGSTAT 101da Autolab ${ }^{\circledR}$ usando $\mathrm{NaCl} 0,01 \mathrm{~mol} \mathrm{~L}^{-1}$ como eletrólito no intervalo de $-0,2$ a $+1,2 \mathrm{~V}$ e sob velocidade de varredura de $20 \mathrm{mV} \mathrm{s}^{-1}$. Os ensaios voltamétricos foram conduzidos em uma célula eletroquímica convencional de três eletrodos contendo o eletrodo de trabalho $\left(\mathrm{Ti} / \mathrm{Ru}_{0.3} \mathrm{Ti}_{0.7} \mathrm{O}_{2}\right)$, um eletrodo de calomelano saturado $\left(\mathrm{Hg} / \mathrm{Hg}_{2} \mathrm{Cl}_{2} \cdot \mathrm{KCl}\right)$ como referência e um eletrodo de platina como auxiliar.

\subsection{Estudos de eletrodegradação}

Os tratamentos eletrocatalíticos foram realizados em reator de vidro de $60 \mathrm{~mL}$ contendo duas placas de eletrodos sob potencial constante fornecido por uma fonte Tektronix PWS2326 DC com leitura simultânea da tensão e corrente com duas saídas variáveis: $0 \sim 32 \mathrm{~V}$ e $0 \sim 6 \mathrm{~A}$ (Figura 2). Os eletrodos usados como anodo e catodo foram representados pelo próprio anodo comercial de $\mathrm{Ti} / \mathrm{Ru}_{0.3} \mathrm{Ti}_{0.7} \mathrm{O}_{2}$, cuja composição nominal é descrita contendo $30 \%$ de $\mathrm{RuO}_{2}$ e $70 \%$ de $\mathrm{TiO}_{2}$ sobre substrato de Ti. Os eletrodos possuem uma área geométrica de $1 \mathrm{~cm}^{2} \mathrm{em}$ cada face e foram distanciados de $2 \mathrm{~cm}$ em posições paralelas e a uma mesma altura no interior do reator para a realização dos testes de degradação eletroquímica. 
Figura 2. Imagem do aparato eletroquímico usado na degradação do azul de metileno.

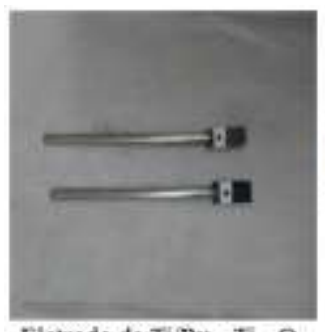

Eletrodo de $\mathrm{Ti} / \mathrm{Ru}_{0,3} \mathrm{TH}_{0} \rightarrow \mathrm{O}_{2}$

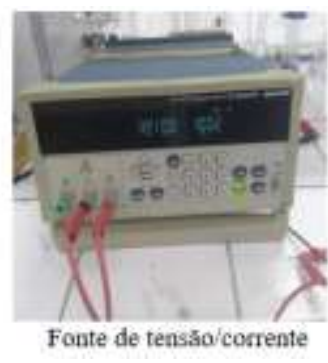

Fonte: Autores.

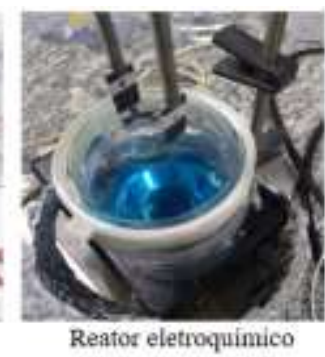

Reator eletroquimico

Os estudos das condições operacionais de eletrooxidação foram executados em pH natural $(\sim 7,0)$ e conduzidos para avaliação do efeito das variáveis de: natureza e concentração do eletrólito $\left(\mathrm{Na}_{2} \mathrm{SO}_{4}\right.$ e $\left.\mathrm{NaCl}\right)$, potencial de eletrólise $(1,5 \mathrm{~V} ; 3,0$ $\mathrm{V}$ e $5,0 \mathrm{~V})$ e concentração de corante $\left(5 \mathrm{mg} \mathrm{L}^{-1} ; 25 \mathrm{mg} \mathrm{L}^{-1} ; 50 \mathrm{mg} \mathrm{L}^{-1}\right.$ e $\left.80 \mathrm{mg} \mathrm{L}^{-1}\right)$.

\subsection{Cinética e monitoramento da degradação}

A cinética de remoção do corante foi descrita segundo um modelo cinético de pseudo primeira ordem (Fayazi \& Ghanei-Motlagh, 2020; Nakamura et al., 2019, Kaur et al., 2018), conforme a Equação 1.

$$
-\frac{d_{c}}{d_{t}}=k_{1} C
$$

Onde: $C$ é a concentração da molécula-alvo na amostra a um dado tempo $t$ e $k_{1}\left(\mathrm{~min}^{-1}\right)$ é a constante cinética de primeira ordem.

A linearização da Equação 1 permite a obtenção direta do valor de $k_{1}$, conforme a Equação 2.

$$
\ln \left(\frac{c}{c_{0}}\right)=-k_{1} t
$$

Onde: $C_{0}$ é a concentração inicial do corante.

O monitoramento da degradação foi realizado usando um espectrofotômetro Thermo Scientific Evolution 60S com célula de quartzo de caminho ótico de $1 \mathrm{~cm}$. Uma curva de calibração para o azul de metileno a $665 \mathrm{~nm}$ foi usada para a determinação da concentração do corante durante os estudos de eletrodegradação.

A eficiência de degradação a temperatura ambiente $\left(25^{\circ} \mathrm{C}\right)$ nos tratamentos eletrooxidativos foi calculada segundo a Equação 3.

$$
\text { Degradação }(\%)=\left(\frac{C_{0}-C_{t}}{c_{0}}\right) \times 100
$$

Onde: $C_{0}$ é a concentração inicial da solução de corante a ser degradada e $C_{t}$ é a concentração da solução de corante $\left(\mathrm{mg} \mathrm{L}^{-1}\right)$ em um tempo de reação. 


\section{Resultados e Discussão}

\subsection{Caracterização do eletrodo de trabalho}

As características superficiais do substrato de Ti e dos filmes de óxidos depositados sobre o anodo de trabalho foram determinadas por microscopia eletrônica de varredura (SEM) com uma ampliação de 1000 x (Figura 3a). A morfologia observada é do tipo "barro rachado", caracterizada por fendas, fissuras e micro rachaduras, que conferem ao eletrodo uma elevada área superficial, conforme relatado por Lanza e Bertazzoli (2002).

Figura 3. Imagem SEM e composição da superfície do eletrodo de $\mathrm{Ti} / \mathrm{Ru}_{0,3} \mathrm{Ti}_{0,7} \mathrm{O}_{2}$.

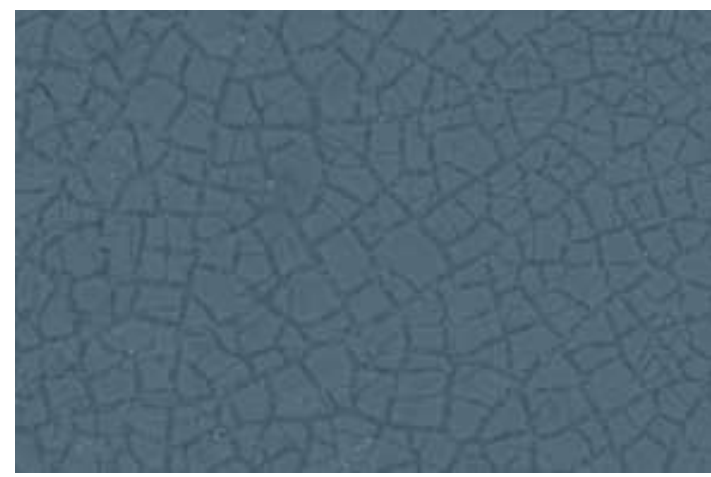

(a)

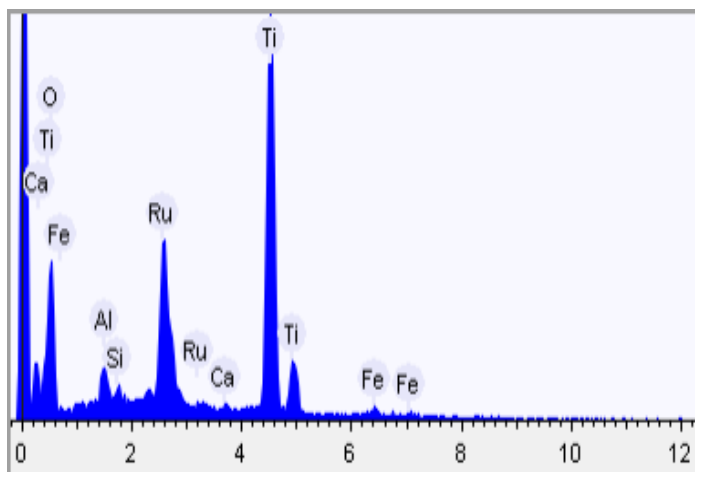

(b)

Fonte: Autores.

A Figura 3b apresenta o perfil por EDX da superfície do anodo de $\mathrm{Ti} / \mathrm{Ru}_{0,3} \mathrm{Ti}_{0,7} \mathrm{O}_{2}$. A composição química do filme precipitado no substrato de titânio mostrou teores de 23,6\% de $\mathrm{RuO}_{2}$ e 71,6\% de $\mathrm{TiO}_{2}$, a qual é próxima da composição teórica (30\% de $\mathrm{RuO}_{2}$ e $70 \%$ de $\mathrm{TiO}_{2}$ ) relatada pelo fabricante. Impurezas metálicas como $\mathrm{Al}, \mathrm{Si}, \mathrm{Ca}$ e Fe foram encontradas em menores quantidades na superfície do eletrodo.

Em termos eletroquímicos, a caracterização do eletrodo de $\mathrm{Ti} / \mathrm{Ru}_{0,3} \mathrm{Ti}_{0,7} \mathrm{O}_{2}$ mostra elevada superposição das curvas voltamétricas (200 ciclos) permitindo observar uma elevada estabilidade das camadas depositadas de óxidos, conforme a Figura 4.

Figura 4. Voltamogramas cíclicos a $25{ }^{\circ} \mathrm{C}$ para o anodo de $\mathrm{Ti} / \mathrm{Ru}_{0,3} \mathrm{Ti}_{0,7} \mathrm{O}_{2}$ na presença (linha preta) e ausência de azul de metileno (linha vermelha) usando $\mathrm{NaCl} 0,01 \mathrm{~mol} \mathrm{~L}^{-1}$ como eletrólito suporte. $C_{0}=5 \mathrm{mg} \mathrm{L}^{-1} ; \mathrm{pH}=6,8$.

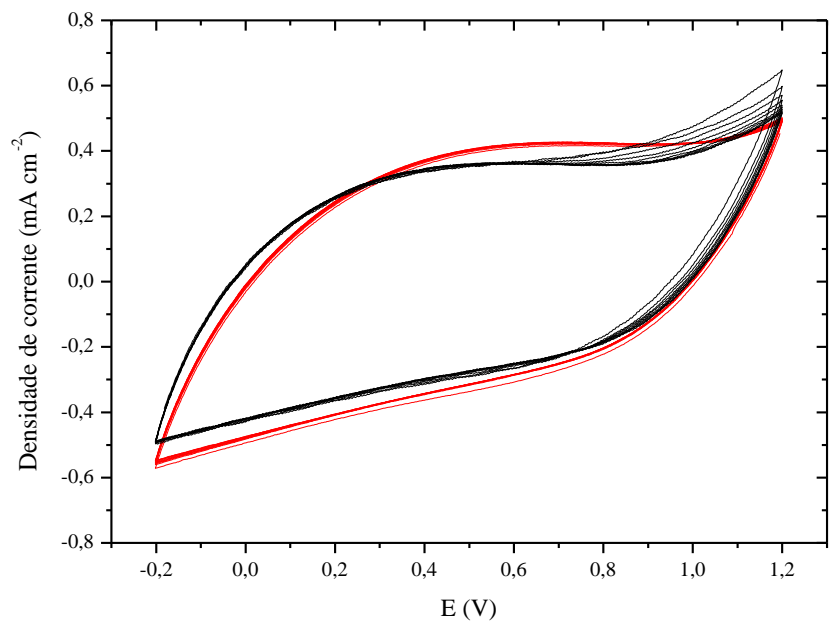

Fonte: Autores. 
Segundo Alves et al. (2004), três processos redox são relacionados ao anodo de $\mathrm{Ti} / \mathrm{Ru}_{0,3} \mathrm{Ti}_{0,7} \mathrm{O}_{2}$ a $\mathrm{pH}=7,0$ : o primeiro ocorre entre $-0,4$ a $0,0 \mathrm{~V}$ corresponde a transição redox $\mathrm{Ru}^{2+} / \mathrm{Ru}^{3+}$, o segundo ocorre entre 0,2 e $0,6 \mathrm{~V}$ e se refere a transição redox $\mathrm{Ru}^{3+} / \mathrm{Ru}^{4+}$ e o terceiro entre 1,0 e $1,3 \mathrm{~V}$ corresponde a reação de desprendimento de oxigênio. Particularmente, não foram observados picos adicionais quando o corante foi adicionado ao meio eletrolítico.

\subsection{Estudos de eletrodegradação}

\subsubsection{Efeito do potencial de eletrólise}

O efeito do potencial sobre a eficiência de degradação do azul de metileno foi inicialmente investigado em um tempo

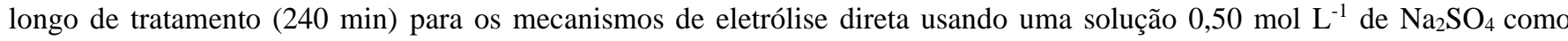
suporte e de oxidação indireta usando um eletrólito misto representado por uma solução $0,01 \mathrm{~mol} \mathrm{~L}^{-1}$ de $\mathrm{Na}_{2} \mathrm{SO}_{4}$ e $0,01 \mathrm{~mol} \mathrm{~L}^{-1}$ de $\mathrm{NaCl}$. Os resultados encontrados estão apresentados na Tabela 1 (mecanismo de oxidação direta) e Figura 5 (mecanismo de oxidação indireta).

Tabela 1. Efeito do potencial na eficiência de eletrodegradação a $25^{\circ} \mathrm{C}$ do azul de metileno $\left(5 \mathrm{mg} \mathrm{L}^{-1}\right)$ sobre anodo de $\mathrm{Ti} / \mathrm{Ru}_{0,3} \mathrm{Ti}_{0,7} \mathrm{O}_{2}$ usando $\mathrm{Na}_{2} \mathrm{SO}_{4}$ 0,50 $\mathrm{mol} \mathrm{L}^{-1}$ como eletrólito suporte.

\begin{tabular}{ccc}
\hline $\begin{array}{c}\text { Potencial de } \\
\text { eletrólise }(\mathrm{V})\end{array}$ & $\begin{array}{c}\text { Densidade de corrente } \\
\left(\mathrm{mA} \mathrm{cm}^{-2}\right)\end{array}$ & $\begin{array}{c}\text { Degradação } \\
(\%)\end{array}$ \\
\hline 1,5 & 10 & 13,2 \\
3,0 & 15 & 40,1 \\
5,0 & 20 & 52,3 \\
\hline
\end{tabular}

Fonte: Autores.

No suporte eletrolítico $\mathrm{Na}_{2} \mathrm{SO}_{4} 0,5 \mathrm{~mol} \mathrm{~L}^{-1}$ as eficiências de degradação foram baixas, o que torna esta condição inviável para uma futura implementação em maior escala. O melhor potencial de eletrólise encontrado foi de $5,0 \mathrm{~V}$ que apresentou 52,3\% de conversão, seguido de 40,1 \% de eficiência para o potencial de $3 \mathrm{~V}$. No potencial de 1,5 V a degradação foi considerada insignificante (13,2\%). Estes resultados mostram que o mecanismo de eletrólise direta (oxidação anódica) a partir da descarga da água com geração de radicais hidroxilos (Equação 4) não foi suficiente para a degradação completa do corante nas condições experimentais aplicadas.

$$
2 \mathrm{H}_{2} \mathrm{O} \rightarrow 2^{\cdot} \mathrm{OH}+2 \mathrm{H}^{+}+2 e^{-}
$$


Figura 5. Efeito do potencial de eletrólise na cinética de eletrodegradação a $25{ }^{\circ} \mathrm{C}$ do azul de metileno $\left(5 \mathrm{mg} \mathrm{L}^{-1}\right)$ sobre anodo de $\mathrm{Ti} / \mathrm{Ru}_{0,3} \mathrm{Ti}_{0,7} \mathrm{O}_{2}$ usando $\mathrm{Na}_{2} \mathrm{SO}_{4} 0,01 \mathrm{~mol} \mathrm{~L}^{-1}$ e $\mathrm{NaCl} 0,01 \mathrm{~mol} \mathrm{~L}{ }^{-1}$ como eletrólito suporte.
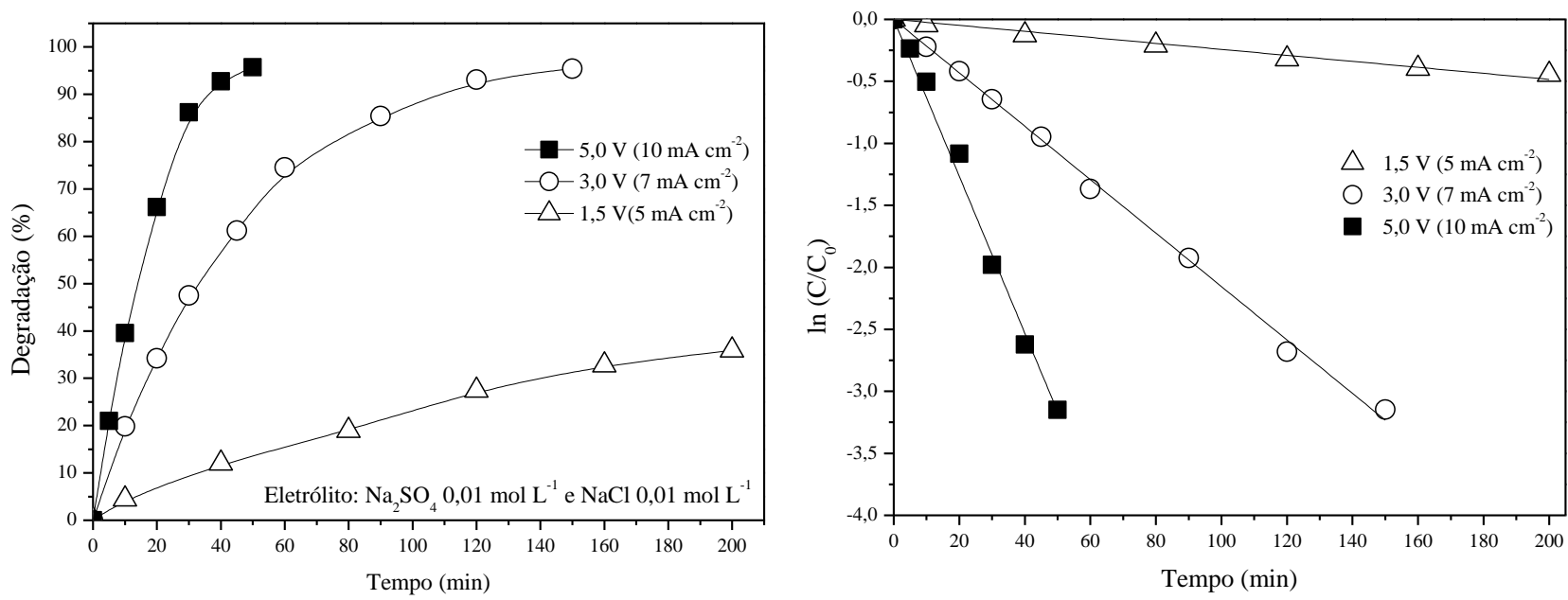

Fonte: Autores.

Diferente do observado na eletrólise direta, a adição de cloreto ao meio de sulfato favorece a degradação pela ação das

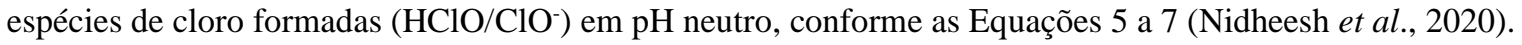

$$
\begin{gathered}
2 \mathrm{Cl}^{-} \rightarrow \mathrm{Cl}_{2}+2 e^{-} \\
\mathrm{Cl}_{2}+\mathrm{H}_{2} \mathrm{O} \rightarrow \mathrm{HClO}+\mathrm{Cl}^{-} \\
\mathrm{HClO} \rightarrow \mathrm{ClO}^{-}+\mathrm{H}^{+}
\end{gathered}
$$

No potencial de 5,0 V e na presença de $\mathrm{Cl}^{-}$a degradação alcançada foi de 95,7\% em 60 min de eletrólise a pH $=6,8$ e temperatura ambiente. Nas mesmas condições de eletrólise indireta sob potenciais de $3,0 \mathrm{~V}$ e $1,5 \mathrm{~V}$ as eficiências de degradação encontradas foram, respectivamente, de 95,4\% em 150 min de reação e de 35,9\% em 200 min de eletrólise. Notadamente, somente os potenciais de 3,0 V e 5,0 V (Figura 5) mostraram-se suficientes para a efetiva degradação do azul de metileno nas condições empregadas, o que pode ser atribuído a da atuação do $\mathrm{HClO}$ formado em pH entre 3 e 8 (Baddouh et al., 2018; Singh et al., 2016).

Os valores da constante cinética de pseudo primeira ordem para o efeito do potencial de eletrólise sobre degradação do azul de metileno estão apresentados na Tabela 2 a seguir.

Tabela 2. Parâmetros cinéticos a $25^{\circ} \mathrm{C}$ para degradação de azul de metileno sob diferentes potenciais de eletrólise. $C_{0}=$ $5 \mathrm{mg} \mathrm{L}{ }^{-1} ; \mathrm{pH}=6,8$ e suporte eletrolítico: $\mathrm{Na}_{2} \mathrm{SO}_{4} 0,01 \mathrm{~mol} \mathrm{~L}^{-1}$ e $\mathrm{NaCl} 0,01 \mathrm{~mol} \mathrm{~L}^{-1}$.

\begin{tabular}{cccc}
\hline $\begin{array}{c}\text { Potencial de } \\
\text { eletrólise }(\mathrm{V})\end{array}$ & $\begin{array}{c}\text { Densidade de } \\
\text { corrente }\left(\mathrm{mA} \mathrm{cm}^{-2}\right)\end{array}$ & $\begin{array}{c}k_{1} \\
\left(\mathrm{~min}^{-1}\right)\end{array}$ & $\mathrm{R}^{2}$ \\
\hline 1,5 & 5,0 & 0,0024 & 0,991 \\
3,0 & 7,0 & 0,0220 & 0,999 \\
5,0 & 8,5 & 0,0630 & 0,996 \\
\hline
\end{tabular}

Fonte: Autores. 
Os resultados cinéticos indicam que as degradações do corante sob diferentes potenciais apresentaram são bem correlacionadas pelo modelo de pseudo primeira ordem aplicado, com valores de $\mathrm{R}^{2}$ superiores a 0,990 . As constantes de velocidades $\left(k_{1}\right)$ para a degradação do azul de metileno aumentaram com o incremento de potencial apresentando valores de 0,0024 $\mathrm{min}^{-1} ; 0,022 \mathrm{~min}^{-1}$ e 0,063 $\mathrm{min}^{-1}$, respectivamente para os potenciais de eletrólise de 1,5; 3,0 e 5,0 V.

No potencial de $1,5 \mathrm{~V}$ a baixa eficiência de degradação de corante e o valor reduzido da constante cinética de primeira ordem podem ser associados a reação competitiva de desprendimento de oxigênio (RDO) que ocorre neste valor de potencial para eletrodos de $\mathrm{Ti} / \mathrm{RuO}_{2}$ (Comninellis \& Chen, 2010). Os valores de $k_{1}$ a 3,0 e 5,0 V são cerca de 9 e 26 vezes maiores que o observado a 1,5 V denotando reações mais favoráveis a partir da formação e atuação das espécies oxidantes cloradas e uma menor interferência da RDO. Adicionalmente, a 5,0 V a cinética reação é ainda quase 3 vezes superior a encontrada a 3,0 V; o que é muito interessante do ponto de vista de futuras aplicações ambientais.

Panizza et al. (2007) avaliaram o efeito da densidade de corrente entre 20 e $60 \mathrm{~mA} \mathrm{~cm}^{-2}$ na degradação eletroquímica a $20^{\circ} \mathrm{C}$ do azul de metileno sobre anodo de Ti/RuTi7 $\mathrm{O}_{2}$ em meio de $\mathrm{Na}_{2} \mathrm{SO}_{4} 0,50 \mathrm{~mol} \mathrm{~L}^{-1}$ e $\mathrm{NaCl} 0,03 \mathrm{~mol} \mathrm{~L}^{-1}\left(1,2 \mathrm{~g} / \mathrm{m}^{3}\right)$. Os autores relataram uma descoloração mais rápida com o incremento da densidade de corrente na faixa de valores empregada. As máximas degradações (100\%) foram obtidas em tempos de aproximadamente $10 \mathrm{~min}, 20 \mathrm{~min}$ e $33 \mathrm{~min}$, respectivamente para densidades de $20 \mathrm{~mA} \mathrm{~cm}{ }^{-2}, 40 \mathrm{~mA} \mathrm{~cm}{ }^{-2}$ e $60 \mathrm{~mA} \mathrm{~cm}^{-2}$. Em termos, o uso de uma menor concentração de $\mathrm{Cl}^{-}\left(0,01 \mathrm{~mol} \mathrm{~L}^{-1}\right)$ e de densidades inferiores de corrente $\left(5\right.$ a $\left.8,5 \mathrm{~mA} \mathrm{~cm}^{-2}\right)$ tornam promissores os resultados obtidos neste trabalho.

Catanho et al. (2006) utilizaram anodo de $\mathrm{Ti} / \mathrm{Ru}_{0.3} \mathrm{Ti}_{0.7} \mathrm{O}_{2}$ no tratamento eletroquímico de corantes têxteis $\left(30 \mathrm{mg} \mathrm{L}{ }^{-1}\right)$ como o preto reativo 5, alaranjado reativo 16 e alaranjado reativo 107 usando $\mathrm{Na}_{2} \mathrm{SO}_{4}$ 0,50 mol L-1 como eletrólito. Os resultados após $3 \mathrm{~h}$ de eletrólise mostraram degradações completas para uma densidade de corrente de $50 \mathrm{~mA} \mathrm{~cm}^{-2}(9,0 \mathrm{~V})$ e uma cinética reacional tipicamente de pseudo primeira ordem, semelhante a observada neste estudo na eletrodegradação do azul de metileno sobre o mesmo eletrodo.

Alaoui et al. (2015) investigaram a degradação de uma solução $40 \mathrm{mg} \mathrm{L}^{-1}$ de azul de metileno em $0,1 \mathrm{~mol} \mathrm{~L}^{-1}$ de $\mathrm{Na}_{2} \mathrm{SO}_{4}$ sobre eletrodo de $\mathrm{Pt} / \mathrm{MnO}_{2}$. Os autores relataram aumento na cinética de degradação de 0,017 $\min ^{-1}$ para $0,025 \mathrm{~min}^{-1}$ com o aumento da densidade de corrente de $3 \mathrm{~mA} \mathrm{~cm}^{-2}$ para $10 \mathrm{~mA} \mathrm{~cm}^{-2}$.

Baddouh et al. (2018) avaliaram o desempenho de um anodo de $\mathrm{Ti} / \mathrm{RuO}_{2}-\mathrm{IrO}_{2}(\mathrm{DSA})$ a $25^{\circ} \mathrm{C}, \mathrm{pH}=6,5 \mathrm{em} 0,1 \mathrm{~mol} \mathrm{~L}$

${ }^{1} \mathrm{Na}_{2} \mathrm{SO}_{4}$ e $0,05 \mathrm{~mol} \mathrm{~L}^{-1} \mathrm{Cl}^{-}$na remoção eletroquímica de Rodamina B (50 mg L $\left.{ }^{-1}\right)$ sob densidades de correntes de 20, 30 e $40 \mathrm{~mA} \mathrm{~cm}{ }^{-2}$. As constantes de velocidades $\left(k_{1}\right)$ da cinética de remoção para o anodo DSA nestas densidades de corrente foram de $0,042 \mathrm{~min}^{-1} ; 0,070 \mathrm{~min}^{-1}$ e $0,079 \mathrm{~min}^{-1}$, respectivamente, as quais tem a mesma ordem de grande das encontradas neste

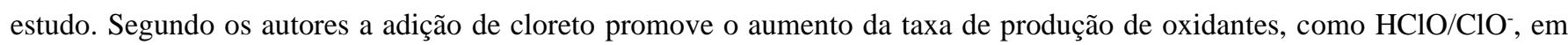
densidades de corrente mais altas levando a eficiências de $100 \%$ de remoção do corante básico a $40 \mathrm{~mA} \mathrm{~cm}$ ch $^{-2}$.

\subsubsection{Efeito do eletrólito suporte}

O efeito do meio eletrolítico a temperatura ambiente sobre a cinética de degradação de azul de metileno a pH natural $(6,8)$ sob potencial de 5,0 V está apresentado na Figura 6 a seguir. 
Figura 6. Efeito do suporte eletrolítico na cinética de degradação a $25^{\circ} \mathrm{C}$ do azul de metileno sobre anodo de $\mathrm{Ti}_{1} / \mathrm{Ru}_{0,3} \mathrm{Ti}_{0,7} \mathrm{O}_{2}$. $C_{0}=5 \mathrm{mg} \mathrm{L}^{-1} ; \mathrm{pH}=6,8$ e potencial de eletrólise: $5,0 \mathrm{~V}$.
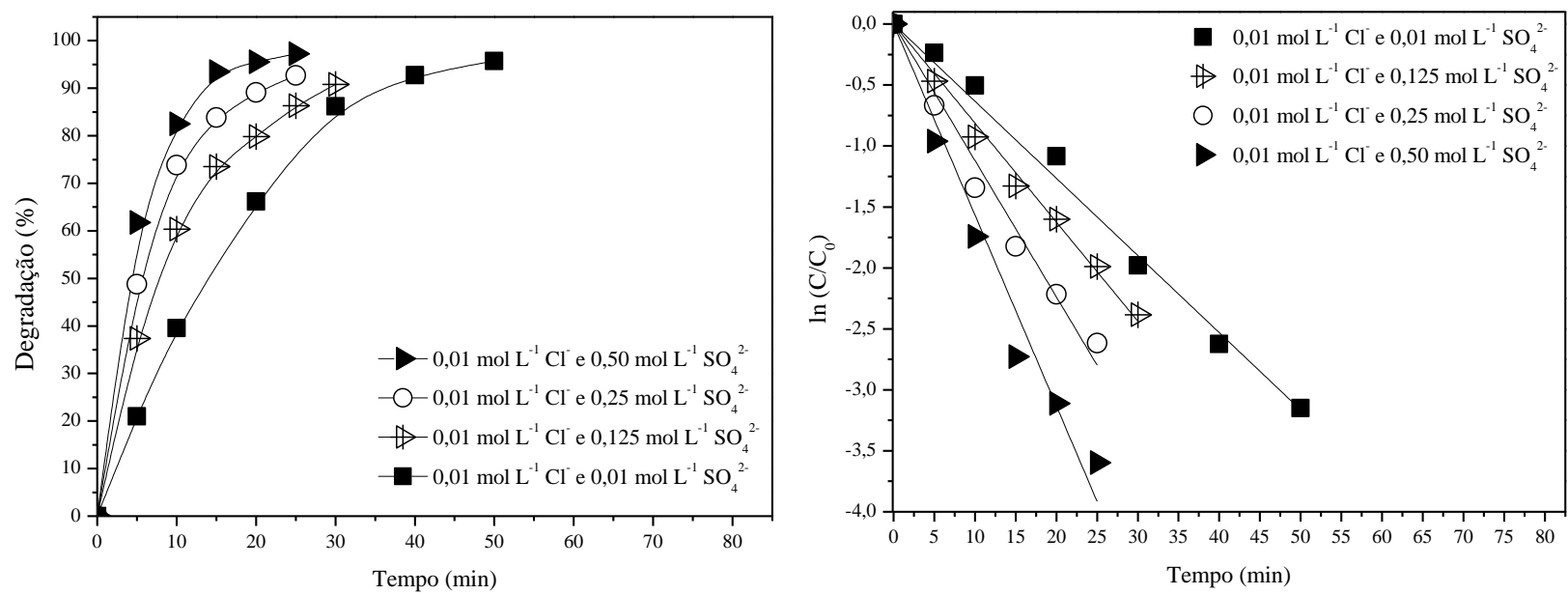

Fonte: Autores.

Na Figura 6 em meio de $\mathrm{Cl}^{-}\left(0,01 \mathrm{~mol} \mathrm{~L}^{-1}\right)$ e $\mathrm{SO}_{4}{ }^{2-}$ (de composição variável) as maiores degradações foram obtidas nas maiores concentrações de sulfato, correspondentes a conversões de 97,5\% e 92,7\% em 0,50 mol L-1 e $0,25 \mathrm{~mol} \mathrm{~L}^{-1}$ de $\mathrm{SO}_{4}{ }^{2-}$ em 25 min de tratamento sob potencial de 5,0 V. A uma concentração de 0,125 mol L-1 de sulfato observa-se uma leve queda de eficiência com degradação máxima de $90,8 \%$ em 30 min de reação. $\mathrm{Na}$ menor concentração de $\mathrm{SO}_{4}{ }^{2-}\left(0,01\right.$ mol $\left.\mathrm{L}^{-1}\right)$ a degradação foi de 86,2\% para 30 min de tratamento e de 95,7 \% em 1 h de reação. Os estudos com eletrólito misto contendo sulfato e cloreto (ambos 0,01 mol L-1) apresentaram degradação elevada (> 90\%) em 60 min de reação, um resultado considerado experimentalmente e ambientalmente satisfatório. A melhor eficiência do sulfato combinado ao cloreto deve-se ao fato da ocorrência de eletrólise indireta, em que o cloreto é oxidado dando origem a espécies altamente oxidantes como o hipoclorito ou ácido hipocloroso, dependendo do pH do meio (Garcia-Segura; Ocon; Chong, 2018; Orts et al., 2020). Em tempo, a concentração de sulfato de $0,01 \mathrm{~mol} \mathrm{~L}^{-1}\left(960 \mathrm{mg} \mathrm{L}^{-1}\right)$ apresenta-se mais atraente dentro de uma perspectiva de menor contaminação ambiental por este ânion. A legislação brasileira no estado de São Paulo (CETESB, 2009) estabelece um limite máximo de descarga de esgotos na rede pública de $1000 \mathrm{mg} \mathrm{L}^{-1}$ para sulfato. O sulfato está associado a problemas de corrosão em águas para o abastecimento industrial e a diminuição da efetividade do tratamento anaeróbio de efluentes industriais, em função de sua redução a sulfeto em condições específicas, o que inibe a atividade bactérias metanogênicas (Callado et al., 2017; Paula Júnior \& Foresti, 2009).

Para o cloreto a Portaria No 5 de 2017 do Ministério da Saúde (Brasil, 2017) estabelece um limite de 250 mg L ${ }^{-1}$, o qual é inferior a concentração de $355 \mathrm{mg} \mathrm{L}^{-1}$ usada neste estudo.

Os valores da constante cinética de primeira ordem para o efeito do suporte eletrolítico sobre degradação do azul de metileno estão apresentados na Tabela 3 a seguir. 
Tabela 3. Parâmetros cinéticos a $25^{\circ} \mathrm{C}$ para degradação de azul de metileno na presença de diferentes meios eletrolíticos. $C_{0}=$ $5 \mathrm{mg} \mathrm{L}^{-1} ; \mathrm{pH}=6,8$ e potencial de eletrólise de 5,0 V.

\begin{tabular}{|c|c|c|}
\hline $\begin{array}{c}\mathrm{NaCl} 0,01 \mathrm{~mol} \mathrm{~L}^{-1}+x \\
\mathrm{~mol} \mathrm{~L}^{-1} \mathrm{Na}_{2} \mathrm{SO}_{4}\end{array}$ & $\begin{array}{c}k_{1} \\
\left(\min ^{-1}\right)\end{array}$ & $\mathrm{R}^{2}$ \\
\hline 0,010 & 0,063 & 0,996 \\
\hline 0,125 & 0,081 & 0,997 \\
\hline 0,250 & 0,112 & 0,992 \\
\hline 0,500 & 0,157 & 0,989 \\
\hline
\end{tabular}

Fonte: Autores.

Na Tabela 3 observa-se que a adição de sulfato ao meio produz valores mais elevados de $k_{1}\left(\mathrm{~min}^{-1}\right)$ como também observado por Tang et al. (2020), os quais relataram que o incremento da adição de $\mathrm{Na}_{2} \mathrm{SO}_{4}$ de 0,025 a 0,10 mol L-1 no meio reacional aumenta a condutividade da solução, favorecendo a transferência de massa no eletrólito e a geração de radicais • $\mathrm{OH}$, o que explica o aumento da taxa de reação na oxidação indireta mantendo constante a concentração de cloreto.

Panizza et al. (2007) verificaram aumento significativo da descoloração, da remoção de matéria orgânica (DQO) e da cinética reacional na eletrodegradação do azul de metileno $\left(80 \mathrm{mg} \mathrm{L}^{-1}\right)$ sobre anodo de Ti/TiRuO a $20 \mathrm{~mA} \mathrm{~cm}^{-2}$ segundo os mecanismos de eletrólise direta $\left(\mathrm{Na}_{2} \mathrm{SO}_{4} 0,50 \mathrm{~mol} \mathrm{~L}^{-1}\right)$ e indireta $\left(\mathrm{NaCl} 0,017 \mathrm{~mol} \mathrm{~L}^{-1}+\mathrm{Na}_{2} \mathrm{SO}_{4} 0,50\right.$ mol L-1 $)$. Na presença de $\mathrm{Na}_{2} \mathrm{SO}_{4}$ 0,50 mol L-1 a redução de DQO foi inferior a 25\% em 120 min de tratamento enquanto após adição de cloreto ao meio a redução de DQO se elevou para cerca de $75 \%$ em 60 min de reação.

Em conformidade com o verificado neste estudo, Salles et al. (2006) observaram um aumento da descoloração de $26 \%$ para $44 \%$ com o incremento da concentração de sulfato entre 0,025 e $0,100 \mathrm{~mol} \mathrm{~L}^{-1}$ para o tratamento de um efluente têxtil real sobre anodo de $\mathrm{Ti} / \mathrm{Ru}_{0.3} \mathrm{Ti}_{0.7} \mathrm{O}_{2}$ a $100 \mathrm{~mA} \mathrm{~cm}{ }^{-2}$ durante 20 min de eletrólise na presença de $0,007 \mathrm{~mol} \mathrm{~L}^{-1}$ de cloreto. O uso de uma concentração $0,250 \mathrm{~mol} \mathrm{~L}^{-1} \mathrm{Na}_{2} \mathrm{SO}_{4}$, por sua vez, mostrou um forte efeito inibidor na redução de cor do efluente.

\subsubsection{Efeito da concentração do corante}

A Figura 7 apresenta o efeito da concentração de corante sobre a eficiência de degradação usando o anodo de $\mathrm{Ti} / \mathrm{Ru}_{0,3} \mathrm{Ti}_{0,7} \mathrm{O}_{2}$.

Figura 7. Efeito da concentração inicial de corante na cinética de degradação a $25{ }^{\circ} \mathrm{C}$ do azul de metileno sobre anodo de $\mathrm{Ti} / \mathrm{Ru}_{0,3} \mathrm{Ti}_{0,7} \mathrm{O}_{2}$. Suporte eletrolítico: $0,01 \mathrm{~mol} \mathrm{~L}^{-1}$ e $\mathrm{NaCl} \mathrm{0,01} \mathrm{mol} \mathrm{L}^{-1}, \mathrm{pH}=6,8$ e potencial de eletrólise: 5,0 V.
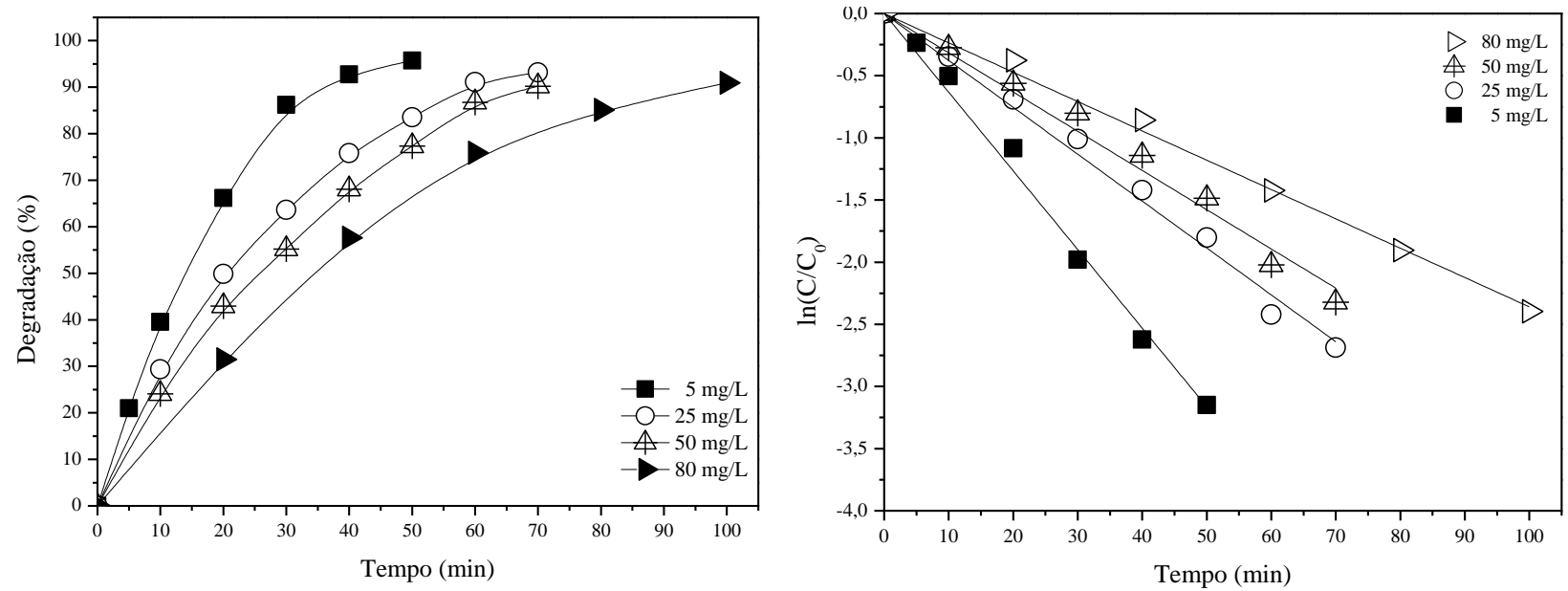

Fonte: Autores. 
$\mathrm{Na}$ Figura 7 observa-se em concentrações inicial de $5 \mathrm{mg} \mathrm{L}^{-1}$ e $25 \mathrm{mg} \mathrm{L}^{-1}$ degradações de 95,7\% e 91,1\% em 60 min de tratamento. Um moderado efeito inibidor da concentração sobre a degradação do corante se apresenta a partir de $50 \mathrm{mg} \mathrm{L}^{-1}$ quando a conversão em 60 min de tratamento se reduz para 86,8\%. A uma concentração de $80 \mathrm{mg} \mathrm{L}^{-1}$ a eficiência de degradação foi ainda menor observando-se apenas 75,9\% de degradação do corante.

Panizza et al. (2007) obtiveram uma eficiência de $100 \%$ na degradação do corante azul de metileno $80 \mathrm{mg} \mathrm{L}^{-1}$ sobre anodo de $\mathrm{Ti} / \mathrm{TiRuO}_{2}$ e densidade de corrente de $20 \mathrm{~mA} \mathrm{~cm}{ }^{-2} \mathrm{em}$ meio eletrolítico contendo cloreto e sulfato. Os resultados obtidos neste trabalho são semelhantes, obtendo-se aproximadamente $91 \%$ de degradação de azul de metileno em 100 min de tratamento.

Samarghandi et al. (2020) avaliaram o efeito da concentração inicial (20,60, 120 e $\left.180 \mathrm{mg} \mathrm{L}^{-1}\right)$ sobre a remoção eletroquímica de azul de metileno em condições ótimas de $\mathrm{pH}(5,75)$; corrente $\left(10 \mathrm{~mA} \mathrm{~cm}{ }^{-2}\right)$ e eletrólito $\left(78,8 \mathrm{mg} \mathrm{L}^{-1} \mathrm{Na}_{2} \mathrm{SO}_{4}\right)$ usando anodos de grafite e de grafite dopado com $\mathrm{PbO}_{2}\left(\mathrm{G} / \beta-\mathrm{PbO}_{2}\right)$. Os autores observaram para o anodo de grafite reduções aproximadas de $68,3 \%$ para $48,2 \%$ com o aumento da concentração na faixa de valores investigadas. No anodo $\mathrm{G} / \beta-\mathrm{PbO}_{2}$ as reduções de eficiência foram de $96,4 \%$ para $84,4 \%$.

Os valores da constante cinética de primeira ordem para o efeito da concentração inicial de corante sobre degradação do azul de metileno estão apresentados na Tabela 4 a seguir.

Tabela 4. Parâmetros cinéticos a $25^{\circ} \mathrm{C}$ para degradação de azul de metileno sob diferentes concentrações iniciais de corante.

Potencial de eletrólise $=5,0 \mathrm{~V} ; \mathrm{pH}=6,8$ e suporte eletrolítico: $\mathrm{Na}_{2} \mathrm{SO}_{4}$ 0,01 mol L-1 e $\mathrm{NaCl}$ 0,01 mol L-1.

\begin{tabular}{ccc}
\hline $\begin{array}{c}\text { Concentração de } \\
\text { corante }\left(\mathrm{mg} \mathrm{L}^{-1}\right)\end{array}$ & $\begin{array}{c}k_{1} \\
\left(\mathrm{~min}^{-1}\right)\end{array}$ & $\mathrm{R}^{2}$ \\
\hline 5 & 0,063 & 0,996 \\
25 & 0,038 & 0,996 \\
50 & 0,032 & 0,993 \\
80 & 0,024 & 0,998 \\
\hline
\end{tabular}

Fonte: Autores.

A Tabela 4 mostra um decréscimo de 1,65 vezes da constante cinética de pseudo primeira ordem $\left(k_{1}\right)$ com o aumento da concentração de $5 \mathrm{mg} \mathrm{L}^{-1}$ para $25 \mathrm{mg} \mathrm{L}^{-1}$ sob moderada redução da eficiência de degradação. Para $80 \mathrm{mg} \mathrm{L}^{-1}$ a velocidade de reação é quase três vezes mais lenta $\left(k_{1}=0,063 \mathrm{~min}^{-1}\right)$ que na mais baixa concentração investigada $\left(k_{1}=0,024 \min ^{-1}\right)$. $\mathrm{O}$ efeito negativo do aumento da concentração na cinética reacional de eletrooxidação do azul de metileno foi também observado por Jawad e Najim (2018). Estes autores usaram anodo de grafite para a remoção de azul de metileno (50, 100, 200 e $400 \mathrm{mg} \mathrm{L}^{-1}$ ) em $\mathrm{Na}_{2} \mathrm{SO}_{4} 0,5 \mathrm{~g} \mathrm{~L}^{-1}$ sob densidade de corrente de $20 \mathrm{~mA} \mathrm{~cm}^{-2}$. A velocidade de degradação do corante diminuiu com o aumento da concentração no meio reacional, variando de $0,036 \mathrm{~min}^{-1} \mathrm{em} 50 \mathrm{mg} \mathrm{L}^{-1}$ para $0,012 \mathrm{~min}^{-1} \mathrm{em} 400 \mathrm{mg} \mathrm{L}^{-1}$.

Samarghandi et al. (2020) descrevem que a baixas concentrações do poluente orgânico, a cinética de degradação eletroquímica é mais rápida do que a taxa de transferência de massa do contaminante para a superfície do anodo; assim a eficiência de remoção da molécula é favorecida. Estes mesmos pesquisadores observaram decréscimo de 1,42 vezes da constante de primeira ordem com o aumento da concentração de $60 \mathrm{mg} \mathrm{L}^{-1}$ para $180 \mathrm{mg} \mathrm{L}^{-1}$ na eletrooxidação sobre ânodo de $\mathrm{G} / \beta-\mathrm{PbO}_{2}$ em meio de sulfato de sódio. 


\subsection{Análise dos produtos formados}

A Figura 8 apresenta os espectros no UV-Vis das amostras de azul de metileno antes e após os tratamentos eletrooxidativo em alguns meios eletrolíticos empregados neste estudo.

Figura 8. Espectros no UV-Vis para a degradação a temperatura ambiente do azul de metileno após tratamento eletroquímico sobre anodo de $\mathrm{Ti} / \mathrm{Ru}_{0,3} \mathrm{Ti}_{0,7} \mathrm{O}_{2}$.

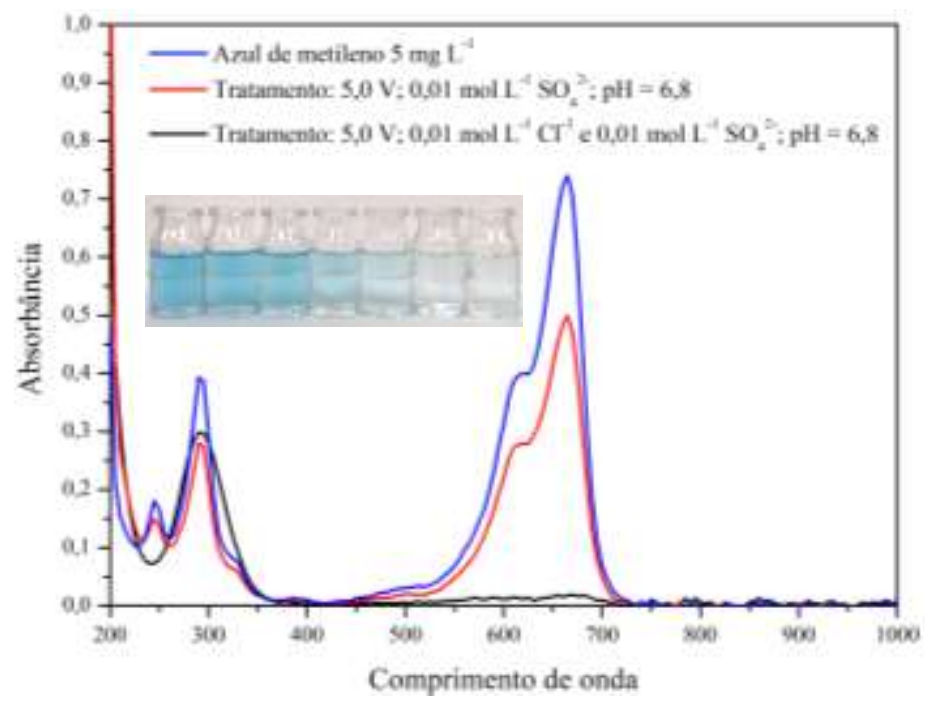

Fonte: Autores.

Na Figura 8, o pico localizado em $665 \mathrm{~nm}$ corresponde ao grupo cromóforo do corante e os picos em $245 \mathrm{~nm}$ e 290 nm são atribuídos aos anéis benzênicos da molécula do corante (Paulino et al., 2015). Após a eletrólise indireta usando o eletrólito misto (cloreto e sulfato) foi observada uma forte redução da banda cromófora via $\mathrm{N}$-desmetilação, conforme observado por Lin et al. (2018). Para o meio contendo somente $\mathrm{Na}_{2} \mathrm{SO}_{4}$ a oxidação direta não foi suficiente para a descoloração do corante, observando-se uma absorbância residual elevada a $665 \mathrm{~nm}$ para a solução eletro-tratada. As bandas a $254 \mathrm{~nm}\left(\pi-\pi^{*}\right)$ e $290 \mathrm{~nm}\left(n-\pi^{*}\right)$ associadas aos anéis aromáticos foram pouco degradadas em todos os tratamentos, o que denota baixa mineralização do corante. Por outro lado, não há evidências direta da formação de outros coprodutos reacionais contendo derivados fenotiazínicos, predominando as reações de N-desmetilação, desaminação e degradação oxidativa (Fadillah et al., 2019, Paulino et al., 2015).

A imagem inserida na Figura 8 mostra a degradação típica do corante sobre tratamento a 5,0 V em meio misto de cloreto e sulfato e anodo de $\mathrm{Ti} / \mathrm{Ru}_{0,3} \mathrm{Ti}_{0,7} \mathrm{O}_{2}$. O perfil de descoloração é intenso após 50 min de reação eletroquímica.

\section{Considerações Finais}

$\mathrm{O}$ anodo comercial $\mathrm{Ti} / \mathrm{Ru}_{0,3} \mathrm{Ti}_{0,7} \mathrm{O}_{2}$ mostrou-se eficiente como material catalítico para o tratamento eletrooxidativo do azul de metileno em meio aquoso e $\mathrm{pH}$ praticamente neutro para uma concentrações entre 5 e $25 \mathrm{mg} \mathrm{L}^{-1}$ usando $\mathrm{NaCl} 0,01 \mathrm{~mol}$ $\mathrm{L}^{-1}$ e $\mathrm{Na}_{2} \mathrm{SO}_{4}$ 0,01 mol L-1 como eletrólito. As eficiências de eletrodegradação foram superiores a $90 \%$ nestas condições operacionais. $\mathrm{O}$ mecanismo de oxidação direta não se mostrou adequado para a oxidação do corante apresentando uma degradação máxima de 52,3\% sob potencial de 5,0 V mesmo empregando-se uma elevada concentração de $\mathrm{Na}_{2} \mathrm{SO}_{4}(0,50$ mol $\left.\mathrm{L}^{-1}\right)$ como suporte eletrolítico. Em geral, o modelo cinético de pseudo primeira ordem mostrou elevado ajuste dos dados experimentais $\left(\mathrm{R}^{2}>0,991\right)$ e a análise preliminar de coprodutos por absorção molecular mostrou um mecanismo de destruição da molécula a partir da clivagem das ligações N-C do grupo cromóforo. 


\section{Referências}

Alaoui, A., El Kacemi, K., El Ass, K., Kitane, S. \& El Bouzidi, S. (2015). Activity of Pt/MnO $\mathrm{Mn}_{2}$ electrode in the electrochemical degradation of methylene blue in aqueous solution. Separation and Purification Technology, 154, 281-289. https://doi.org/10.1016/j.seppur.2015.09.049.

Alves, P. D. P., Spagnol, M., Tremiliosi-Filho, G. \& Andrade, A. R. (2004). Investigation of the influence of the anode composition of DAS-type eletrodes on the electrocatalytic oxidation on phenol in neutron medium. Journal Brazilian Chemical Society, 15, 626-634. https://doi.org/10.1590/S010350532004000500003.

Baddouh, A., Bessegato, G. G., Rguiti, M. M., El Ibrahimi, B., Bazzi, L., Hilali, M. \& Zanoni, M. V. B. (2018). Electrochemical decolorization of Rhodamine B dye: Influence of anode material, chloride concentration and current density. Journal of Environmental Chemical Engineering, 6(2), 2041-2047. https://doi.org/10.1016/j.jece.2018.03.007.

Brasil. Ministério da Saúde. (2017). Portaria de consolidação $n \quad{ }^{\circ} 5$, de 28 de 2017. https://portalarquivos2.saude.gov.br/images/pdf/2018/marco/29/PRC-5-Portaria-de-Consolida----o-n---5--de-28-de-setembro-de-2017.pdf

Callado, N. H., Damianovic, M. H. Z. \& Foresti, E. (2017). Influência da razão DQO/[SO $\left.{ }_{4}^{-2}\right]$ e da concentração de Na ${ }^{+}$na remoção de matéria orgânica e sulfato em reator UASB. Engenharia Sanitária Ambiental, 22(2), 381-390, 2017. https://doi.org/10.1590/s1413-41522016140811.

Catanho, M., Malpass, G. R. P. \& Motheo, A. D. J. (2006). Evaluation of electrochemical and photoelectrochemical methods for the degradation of three textile dyes. Quimica Nova, 29(5), 983-989. https://doi.org/10.1590/s0100-40422006000500018.

Companhia de Tecnologia Ambiental do Estado de São Paulo - CETESB. (2009). Apêndice D: Significado Ambiental e Sanitário das Variáveis de Qualidade. São Paulo: CETESB.

Collivignarelli, M. C., Abbà, A., Miino, M. C. \& Damiani, S. (2019). Treatments for color removal from wastewater: State of the art. Journal of Environmental Management, 236, 727-745. https://doi.org/10.1016/j.jenvman.2018.11.094.

Comninellis, C. \& Chen, G. (2010). Electrochemistry for the Environment. Springer-Verlag.

Cruz-Díaz, M. R., Rivero, E. P., Rodríguez, F. A. \& Domínguez-Bautista, R. (2018). Experimental study and mathematical modeling of the electrochemical degradation of dyeing wastewaters in presence of chloride ion with dimensional stable anodes (DSA) of expanded meshes in a FM01-LC reactor. Electrochimica Acta, 260, 726-737. https://doi10.1016/j.electacta.2017.12.025.

Fadillah, G., Saleh, T. A., Wahyuningsih, S., Putri, E. N. K. \& Febrianastuti, S. (2019). Electrochemical removal of methylene blue using alginate-modified graphene adsorbents. Chemical Engineering Journal, 378, 122140-122150. https://doi.org/10.1016/j.cej.2019.122140.

Fayazi, M. \& Ghanei-Motlagh, M. (2020). Electrochemical mineralization of methylene blue dye using electro-Fenton oxidation catalyzed by a nove sepiolite/pyrite nanocomposite. International Journal of Environmental Science and Technology, 17(11), 4541-4548. https://doi: 10.1007/s13762-020-02749-2.

Garcia-Segura, S., Ocon, J. D. \& Chong, M. N. (2018). Electrochemical oxidation remediation of real wastewater effluents - A review. Process Safety and Environmental Protection, 113, 48-67. https://doi.org/10.1016/j.psep.2017.09.014.

Gupta, A. K., Pal, A. \& Sahoo, C. (2006). Photocatalytic degradation of a mixture of Crystal Violet (Basic Violet 3) and Methyl Red dye in aqueous suspensions using $\mathrm{AgC}$ doped $\mathrm{TiO}_{2}$. Dyes and Pigments, 69, 224-232. http://dx.doi.org/10.1016/j.dyepig.2005.04.001

Indu, M. S., Gupta, A. K. \& Sahoo, C. (2014). Electrochemical oxidation of methylene blue using lead acid battery anode. APCBEE Procedia, 9, 70-74. https://doi.org/10.1016/j.apcbee.2014.01.013.

Jawad, N. H. \& Najim, S. T. (2018). Removal of methylene blue by direct electrochemical oxidation method using a graphite anode. Materials Science and Engineering, 454, 1-10. https://doi.org/10.1088/1757-899X/454/1/012023.

Kaur, P., Sangal, V. K. \& Kushwaha, J. P. (2019). Parametric study of electro-Fenton treatment for real textile wastewater, disposal study and its cost analysis. International Journal of Environmental Science and Technology, 16(2), 801-810. https://doi.org/10.1007/s13762-018-1696-9.

Krstić, V. \& Pešovski, B. (2019). Reviews the research on some dimensionally stable anodes (DSA) based on titanium. Hydrometallurgy, 185, 71-75. https://doi.org/10.1016/j.hydromet.2019.01.018.

Lanza, M. R. V. \& Bertazzoli, R. (2002). Selection of a commercial anode oxide coating for electro-oxidation of cyanide. Journal of the Brazilian Chemical Society, 13, 345-351. https://doi.org/10.1590/S0103-50532002000300009.

Lin, J., Luo, Z., Liu, J. \& Li, P. (2018). Photocatalytic degradation of methylene blue in aqueous solution by using $\mathrm{ZnO}^{-S n O} \mathrm{O}_{2}$ nanocomposites. Materials Science in Semiconductor Processing, 87(20), 24-31. https://doi.org/10.1016/j.mssp.2018.07.003.

Li, Y., Du, Q., Liu T., Sun J., Wang, Y., Wu, S., Wang, Z., Xia, Y. \& Xia, L. (2013). Methylene blue adsorption on graphene oxide/calcium alginate composites. Carbohydrate Polymers, 95, 501-507. http://dx.doi.org/10.1016/j.carbpol.2013.01.094.

Martínez-Huitle, C. A. \& Panizza, M. (2018). Electrochemical oxidation of organic pollutants for wastewater treatment. Current Opinion in Electrochemistry, 11(1), 62-71. https://doi.org/10.1016/j.coelec.2018.07.010.

Nakamura, K. C., Guimarães, L. S., Magdalena, A. G., Angelo, A. C. D., De Andrade, A. R., Garcia-Segura, S. \& Pipi, A. R. F. (2019). Electrochemicallydriven mineralization of reactive blue 4 cotton dye: On the role of in situ generated oxidants. Journal of Electroanalytical Chemistry, 840, 415-422. https://doi.org/10.1016/j.jelechem.2019.04.016.

Nidheesh, P. V., Kumar, A., Babu, D. S., Scaria, J. \& Kumar, M. S. (2020). Treatment of mixed industrial wastewater by electrocoagulation and indirect electrochemical oxidation. Chemosphere, 251, 126437-126447. https://doi.org/10.1016/j.chemosphere.2020.126437. 
Research, Society and Development, v. 10, n. 5, e35510514918, 2021

(CC BY 4.0) | ISSN 2525-3409 | DOI: http://dx.doi.org/10.33448/rsd-v10i5.14918

Oliveira, L. G., Fernandes, F. H., Mesquita, W. D., Junior, M. G., Santos, M. R. C. \& Gurgel, M. F. C. (2020). Uma revisão do uso de processos oxidativos avançados para descoloração de águas residuais de efluentes. Revista Processos Químicos, 13(26), 105-112. https://doi.org/10.19142/rpq.v13i26.546.

Orts, F., Bonastre, J., Fernández, F. \& Cases, F. (2020). Effect of chloride on the one step electrochemical treatment of an industrial textile wastewater with tin dioxide anodes. The case of trichromy procion HEXL. Chemosphere, 245, 125396-125403. https://doi.org/10.1016/j.chemosphere.2019.125396.

Paula Júnior., D. R. \& Foresti, E. (2009). Sulfide toxicity kinetics of a UASB reactor. Brazilian Journal of Chemical Engineering, 26(4), 669-675. https://doi.org/10.1590/S0104-66322009000400005.

Paulino, T. R. S., Araújo, R. S. \& Salgado, B. C. B. (2015). Estudo de oxidação avançada de corantes básicos via reação Fenton (Fe $\left.{ }^{2+} / \mathrm{H}_{2} \mathrm{O}_{2}\right)$. Engenharia Sanitaria e Ambiental, 20(3), 347-352. https://doi.org/10.1590/S1413-41522015020000111627.

Salles, P. T. F., Pelegrine, N. N. B \& Pelegrine, R. T. (2006). Tratamento eletroquímico de efluente industrial contendo corantes reativos. Engenharia Ambiental, 3(2), 25-40

Samarghandi, M. R., Dargahi, A., Shabanloo, A., Nasab, H. Z., Vaziri, Y. \& Ansari, A. (2020). Electrochemical degradation of methylene blue dye using a graphite doped $\mathrm{PbO}_{2}$ anode: Optimization of operational parameters, degradation pathway and improving the biodegradability of textile wastewater. Arabian Journal of Chemistry, 13(8), 6847-6864. https://doi.org/10.1016/j.arabjc.2020.06.038.

Santos, D. H. S., Duarte, J. L. S., Tavares, M. G. R., Tavares, M. G., Friedrich, L. C., Meili, L., Pimentel, W. R. O, Tonholo, J. \& Zanta, C. L. P. S. (2020). Electrochemical degradation and toxicity evaluation of reactive dyes mixture and real textile effluent over DSA ${ }^{\circledR}$ electrodes. Chemical Engineering and Processing - Process Intensification, 153, 107940-107951. https://doi.org/10.1016/j.cep.2020.107940.

Singh, S., Lo, S. L., Srivastava, V. C. \& Hiwarkar, A. D. (2016). Comparative study of electrochemical oxidation for dye degradation: Parametric optimization and mechanism identification. Journal of Environmental Chemical Engineering, 4(3), 2911-2921. https://doi.org/10.1016/j.jece.2016.05.036.

Tang, Y., He, D., Guo, Y., Qu, W., Shang, J., Zhou, L., Pan, R. \& Dong, W. (2020). Electrochemical oxidative degradation of X-6G dye by boron-doped diamond anodes: Effect of operating parameters. Chemosphere, 258, 127368-127377. https://doi.org/10.1016/j.chemosphere.2020.127368.

Yaseen, D. A. \& Scholz, M. (2019). Textile dye wastewater characteristics and constituents of synthetic effluents: A critical review. International Journal of Environmental Science and Technology, 16, 1193-1226. https://doi.org/10.1007/s13762-018-2130-z. 